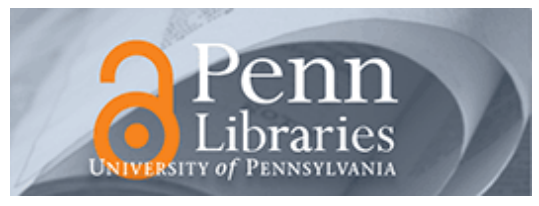

University of Pennsylvania

ScholarlyCommons

Management Papers

Wharton Faculty Research

6-1-2010

\title{
Organizational Routines Development and New Venture Performance
}

David H. Hsu

University of Pennsylvania

Alessandro Marino

University of Pennsylvania

Follow this and additional works at: https://repository.upenn.edu/mgmt_papers

Part of the Business Administration, Management, and Operations Commons, and the Organizational Behavior and Theory Commons

Recommended Citation

Hsu, D. H., \& Marino, A. (2010). Organizational Routines Development and New Venture Performance. Social Science Research Network, http://dx.doi.org/10.2139/ssrn.1618829

This paper is posted at ScholarlyCommons. https://repository.upenn.edu/mgmt_papers/23

For more information, please contact repository@pobox.upenn.edu. 


\title{
Organizational Routines Development and New Venture Performance
}

\begin{abstract}
To better understand how entrepreneurial ventures vary as they evolve, we introduce and develop the concept of an organizational routine in a prototypical state, a protoroutine. Protoroutines allow experienced new ventures (but not inexperienced start-ups) to economize on decision-making and execution time in problem solving by drawing from an inventory of prior solutions to challenges. Protoroutines are not, however, tailored to the challenge at hand. We embed protoroutines into a simulation-based model featuring agents with differing decision-making speeds and abilities of exploring more distant solutions, two parameters influenced by founding team characteristics. Search speed and distance are typically traded off against each other at the team design level. Protoroutines may therefore be particularly helpful in organizational contexts in which it is optimal to have both search speed and distance. We characterize the organizational contextual configurations along the dimensions of environmental turbulence and decision complexity in which protoroutines, search speed, and search distance are associated with elevated (and dampened) organizational performance. One important conclusion is that decision-making speed can be a valuable organizational resource across organizational environments. Overall, our agent-based model and simulation results deepen our understanding of how and with what performance consequence new ventures develop.
\end{abstract}

\section{Keywords}

organizational routine formation, founding team characteristics, new venture performance, agent-based simulation model

\section{Disciplines}

Business Administration, Management, and Operations | Organizational Behavior and Theory 


\title{
Organizational Routines Development and New Venture Performance
}

\author{
David H. Hsu and Alessandro Marino*
}

June 2010

\begin{abstract}
To better understand how entrepreneurial ventures vary as they evolve, we introduce and develop the concept of an organizational routine in a prototypical state, a protoroutine. Protoroutines allow experienced new ventures (but not inexperienced start-ups) to economize on decision-making and execution time in problem solving by drawing from an inventory of prior solutions to challenges. Protoroutines are not, however, tailored to the challenge at hand. We embed protoroutines into a simulation-based model featuring agents with differing decision-making speeds and abilities of exploring more distant solutions, two parameters influenced by founding team characteristics. Search speed and distance are typically traded off against each other at the team design level. Protoroutines may therefore be particularly helpful in organizational contexts in which it is optimal to have both search speed and distance. We characterize the organizational contextual configurations along the dimensions of environmental turbulence and decision complexity in which protoroutines, search speed, and search distance are associated with elevated (and dampened) organizational performance. One important conclusion is that decision-making speed can be a valuable organizational resource across organizational environments. Overall, our agent-based model and simulation results deepen our understanding of how and with what performance consequence new ventures develop.
\end{abstract}

Keywords: organizational routine formation; founding team characteristics; new venture performance; agent-based simulation model.

${ }^{*}$ The Wharton School, University of Pennsylvania, 3620 Locust Walk, 2000 Steinberg Hall-Dietrich Hall, Philadelphia, PA 19104. We thank Dan Levinthal for numerous helpful discussions, Marco Valente for his technical support in developing the simulation code, and Olivier Chatain, Gary Dushnitsky, Lori Rosenkopf, Nicolaj Siggelkow, and seminar participants at Drexel University and the 2010 Atlanta Competitive Advantage Conference for their comments. The Mack Center for Technological Innovation at Wharton and the Alfred P. Sloan Foundation supported this research. 


\section{Introduction}

We have little understanding of how start-ups vary as they develop. In part, this gap results from the difficulty of defining when a start-up "graduates" to a more established status. Consequently, the entrepreneurship literature tends to consider a bimodal distribution of settings. One set of studies considers brand new start-ups and early-stage challenges such as venture idea appropriation, recruiting, setting strategy, and resource assembly. Another set of studies examines relatively latestage phenomena such as corporate governance before and after an initial public offering and the long-run performance of venture capital funded ventures. While this is a gross characterization of the literature in that some studies have examined intermediate stages of venture development such as the financial contracting consequences of attaining revenue-positive status (Kaplan and Stromberg 2003) and the evolution of human resource management systems (Baron et al. 1996), our aim is to explicitly study the new venture evolution process.

We do so by introducing the concept of a protoroutine, an organizational routine that is not yet perfected, and is therefore in a prototype stage of development. Like routines, which can be enacted in response to a given stimulus or task (e.g., an employee recruiting routine), protoroutines allow firms to economize on decision-making time (Nelson and Winter 1982). Protoroutines differ in their usage relative to routines, however, in that they are not tailored to the task or challenge at hand. The concept of protoroutines accords well with the sparse literature examining intermediate stages of venture growth. Emergent (yet unperfected) organizational processes are likely associated with, for example, being cash-flow positive or developing a formal human resource management system. Such processes are available to experienced new firms but do not yet exist in completely new ventures.

We study protoroutines in the context of ventures that differ in their founding team characteristics and in the organizational environments in which they operate, allowing us to address the research question: what is the effect of emergent organizational routines on venture performance 
given different founding team and environmental characteristics? To motivate our agent-based simulation model and method, we first discuss the literature on organizational resources afforded by founding team characteristics, culminating in the proposition that there might be a team design tradeoff in achieving both decision-making speed and search distance. Since protoroutines emerge partly as a result of organizational choice in pace of development and serves the function of economizing on decision-making speed, protoroutines serve two purposes in our study. Protoroutines both describe an organizational process associated with venture development and serve as an organizational instrument that may be useful, particularly in the case of functional tradeoffs at the founding team design level. Our simulation seeks to understand the organizational environmental conditions under which protoroutines bolster or dampen venture performance.

One of the earliest and potentially most important challenges for founders of entrepreneurial ventures is assembling a founding team. A multitude of factors may potentially guide team composition, and these factors may span both current and anticipated venture needs. ${ }^{1}$ A robust interdisciplinary literature investigates the relationship between founder (and top management) team characteristics and venture performance. On the one hand, some studies have found that teams with varied functional backgrounds and perspectives are generally more able to explore distant problem-solving terrain relative to more homogenous teams (Eisenhardt and Schoonhoven 1990; Ancona and Caldwell 1992; Smith et al. 1994; Hong and Page 2004; Beckman 2006). Diverse teams, however, tend to be less cohesive (Schweiger et al. 1986), worse in communicating and managing conflict (Smith et al. 1994), and slower at decision-making (Virany et al. 1992; Geletkanycz and Hambrick 1997). Uniform teams, on the other hand, tend to be faster in decision-making

\footnotetext{
${ }^{1}$ While altering team composition might be one way of addressing the evolving needs of the organization, a body of literature suggests powerful founder "imprinting" effects wherein policies, procedures, and organizational culture established at organizational birth can have long-lived effects on corporate strategy, human resource management, and top management teams (Stinchcombe 1965; Boeker 1989; Eisenhardt and Schoonhoven 1990; Baron et al. 1996). We take founder imprinting as a starting point by recognizing that founding team composition can exert long-term effects that can shape venture performance. However, in keeping with the desire to further understand new venture evolution, we overlay protoroutine development at the organizational level to better understand the process of venture development.
} 
(Murray 1989) and have higher mutual understanding and trust (Stinchcombe 1965), but tend to underperform diverse teams in innovating (Bantel and Jackson 1989).

The general findings from the team design literature are that uniform teams are faster in decision-making and execution speed while diverse teams are better at generating more distant or novel problem-solving ideas. A simple theory of optimal founding team composition would therefore be to form teams that are both homogenous and diverse along different dimensions (e.g., common prior employer but varied functional backgrounds). Two studies suggest that this approach is sensible. Eisenhardt and Schoonhoven (1990) found that successful newly-founded US semiconductor firms combine healthy conflict (associated with founders' diverse industry experience) with fast decision-making (linked to founders' joint work experience). Beckman (2006) analyzed 170 young high-technology firms in Silicon Valley and concluded that founding teams with both diversity and common prior company affiliations experienced superior performance. The literature, however, does not give guidance on the organizational contexts when it is preferable to have elements of both team diversity and uniformity.

We consider the effect of decision-making speed (which is related to team characteristics and protoroutine use) and search distance (related to team characteristics) given two non-mutually exclusive aspects of the organizational environment, turbulence and complexity. Turbulence is the rate of external environmental change across time periods and can incorporate elements of technology and/or competitive landscape shifts. Complexity is a function of decision interdependence. A low complexity environment occurs when the payoff to one decision is not dependent on another decision made within the organization, with high complexity characterized by highly coupled decision choices.

Empirically assessing the relationship between protoroutines and venture performance given different founding team and organizational environmental characteristics is challenging because it is difficult to assemble a dataset which simultaneously and comprehensively incorporates variation 
in all the necessary elements. Moreover, we believe that our focal domain will benefit from a simulation analysis because it involves some empirical or analytic grounding but is limited by weak conceptualization (as Murray (1989) and Smith et al. (1994) acknowledge), particularly in the domain of intermediate venture development. Such a context of pre-existing simple, but not comprehensive, theory is ripe for simulation-based analysis (Davis et al. 2007).

Our main simulation results can be briefly described as follows. First, decision-making speed is positively associated with organizational performance for all agents, regardless of degree of environmental turbulence and decision complexity. The performance boost of greater decision-making speed is higher for inexperienced start-ups relative to experienced new ventures, however, suggesting that faster speed can partially offset inexperienced agents' inability to use protoroutines. In addition, experienced agents' performance suffers in the face of environmental turbulence and is bolstered by decision complexity, while inexperienced ventures' performance is largely invariant to environmental turbulence. With low decision complexity, a low search distance is best, but as complexity increases, teams' ability to search at greater ranges yields superior performance.

The remaining sections of the paper develop the conceptual foundations of protoroutines before describing our agent-based simulation model and the results. A discussion section concludes the paper.

\section{Routine Formation in New Ventures}

Organizational routines, which are characterized in the literature as semi-automatic patterns of actions that emerge from collective and coordinated interactions among individuals (Nelson and Winter 1982), are central to the evolutionary theory of the firm. Yet while we have a general understanding of factors instigating changes in routines such as crises (Gersick and Hackman 1990; Feldman and Pentland 2003), we know little about the process by which routines are formed and the consequences of using routines in prototype form. We suspect this stems from the shortfall 
in the literature in articulating the organizational processes by which new ventures develop. As a prelude to describing our simulation model, we compare and contrast protoroutines with established organizational routines (which have received the lion's share of attention in the literature) in an effort to both improve protoroutine conceptualization and motivate our modeling of the concept.

A protoroutine is a tentative solution to a problem, and is drawn from an organization's inventory of prior solutions. The latter property draws on the notion that routines more generally embody successful solutions to problems solved by the organization in the past (Paoli and Prencipe 2003), while the former property is inspired by Nelson and Winter's (1982: 130-131) conceptualization of routine formation arising from new, "trial-and-error" combinations of existing routines. As with routines, protoroutines allow the firm to economize on cognitive effort (and can therefore save decision-processing time). Protoroutines, however, differ from established organizational routines in both their usage and storage.

Established routines are used almost without conscious volition (Nelson and Winter 1982: 75) when organizations encounter a situation of a particular type as a result of repeated experience with the stimulus. In contrast, protoroutines are selected and implemented after a deliberate decisionmaking process due to ventures' limited interaction with a given stimulus. Consistent with the notion of learning curves in which learning is initially slow due to response-based experimentation, protoroutines are tentative solutions to challenges that are adjusted over time. As a result, protoroutines are volatile, and are more likely to either disappear after a few applications or to be radically updated. Protoroutines may be developed over time in ventures both as a result of simple operating experience as well as via conscious business policy, for example through codification. Well-tested and robust protoroutines will "graduate" to become organizational routines.

Protoroutines also tend not to be tailored to specific tasks. While more established organizations develop a portfolio of coordinated behavior that may allow them to adapt to specific contingencies and address particular problems, younger firms do not have such an inventory of rou- 
tines. Due to their limited experience, new ventures have only a superficial understanding of the causal correspondence between protoroutines and their effects. As a result, younger organizations will be more flexible in using and testing protoroutines on a wider range of situations.

There is also a difference in the organizational storage of routines as compared to protoroutines. Whereas the locus of memory of routines is embedded within the organization as a whole (Nelson and Winter 1982), protoroutine storage is more at the individual founder or founding team

level. Protoroutines are therefore more sensitive to changes in founding team composition, while established routine effectiveness may be stable even with full turnover of individuals or executives associated with a function.

\section{Agent-Based Simulation Model}

To examine the performance impact of protoroutine development given different founding team characteristics and organizational environments, we describe our agent-based simulation model. We first define the task environment and then describe the agents' characteristics.

\subsection{Defining the Task Environment}

We adopt a problem-based approach according to which agents solve a sequence of challenges in the simulation. Each challenge has a structure of a conventional NK fitness landscape (Kauffman 1993), which is the set of all possible $2^{N}$ binary strings of length $N$ and their associated fitness values. Each string represents the $N$ decisions that agents may make. The fitness value is the performance associated with each string of binary bits, and is generated by assigning a contribution value to each bit of the binary string. The overall fitness value associated with an $N$-vector of decisions is then the average of all the contribution values. Because of the presence of possible interactions among the $N$ bits, changes in specific choices might indirectly affect other choices. The higher the number of interactions among the choices, the more rugged the performance landscape will be (the higher the 
number of performance levels the firm might achieve). Conversely, low-interaction landscapes will be characterized by a lower number of local peaks. The number of interactions among each of the $N$ decisions is determined by the parameter $K$ (in the manner proposed by Kauffman (1993), which aims to capture interdependencies among a firm's decisions and how they relate to environmental complexity, following the concept advanced by Simon (1962)). As a result, each decision within the $N$-dimensional vector of decisions will be associated with a contribution value, which is a function of how the binary decision and the other elements that affect that particular decision are chosen. For each possible combination of decisions and the other decisions that affect it, a contribution value is defined by a random draw from a uniform distribution $\{0,1\}$.

The distinct NK landscapes that define the sequence of challenges that the agents face over the simulation are generated by the following mechanism. First, an arbitrary initial NK fitness landscape constitutes challenge 0. Altering the fitness contributions of the landscape preceding it generates the subsequent landscape, and applying this process recursively generates the remaining challenges. Formally, let $f c_{0}$ be the set of all the possible distinct fitness contributions existing in the original NK landscape (challenge 0 ). For challenge $m$ and the set of the distinct fitness contributions, $f c_{m}=f c_{m-1}+R N D[-R W ;+R W] .{ }^{2}$ This function assigns a new set of fitness contributions by altering fitness contributions in the prior challenge according to a random walk of range $R W$. The NK landscapes therefore shift across challenges, with modified peak topology while maintaining complexity structure $K$. The parameter $R W$ allows us to model differences in turbulence across industries, with higher values of this parameter reducing the degree of landscape correlation across challenges. This landscape discontinuity is a key feature of environmental turbulence (Murray 1989; Keck 1997; Carpenter and Fredrickson 2001). Figure 1 summarizes this overall task environment.

To map the task environment to the new venture development process, consider the following example. Ventures confront correlated challenges over time. For each challenge, the founding team faces a string of binary decisions, $N$ (which comprise a policy). Such decisions may span, for

\footnotetext{
${ }^{2}$ Note that the number of distinct fitness contributions in a given NK landscape equals $N * 2^{(1+K)}$.
} 
example, organizational design and level of vertical integration (e.g., whether to outsource manufacturing, whether to backward integrate into research and development, and whether to partner with a given industry incumbent). Other decisions may relate to human resource management (e.g., whether to allow dedicated time for employees to conduct undirected activities, whether to use performance-based compensation schemes, and whether to replace the chief executive officer of the venture). Still other decisions may relate to revenues and funding (e.g., whether to offer a volume discount to a particular chain store, whether to raise venture capital funding, and whether to utilize an advertising-based revenue model). The parameter $K$ refers to the level of interdependence of the $N$ decisions. The productivity of one human resource management decision, for example, may importantly depend on a complementary human resource decision (Ichniowski et al. 1997) or to decisions made with respect to information technology (Bresnahan et al. 2002). If the $N$ decisions are highly coupled with respect to overall efficacy, ventures will face high decision complexity.

To gain more insight on the properties of the family of NK landscapes generated by this mechanism, we analyze the evolution of local peak distribution across challenges. A local peak is a binary string whose associated fitness cannot be improved by any possible 1-bit mutation of its elements. The number and distributions of the local peaks characterize the topology of each NK landscape (Kauffman 1993). In an unreported analysis we calculated the average number of local peaks observed across 300 challenges generated by the mechanism described above. We analyzed three distinct levels of complexity $(K=\{0,2,5\})$ and two levels of random walk ranges $(R W=\{0.01$, $0.05\})$. As expected, the average number of local peaks increases with $K$ and stabilize to 1 in case of single-peaked worlds $(K=0)$ (Kauffman 1993). The analysis confirms that our method preserves the complexity structure of the sequence of challenges in that the average number of local peaks tends to fluctuate in a relatively narrow bandwidth. This bandwidth increases with higher ranges of the random walk and so in the presence of higher turbulence adjacent challenges composing the sequence tend to be less correlated. The graphs reported in Figure 2 provide further support. We 
first analyze the fraction of local peaks coincident with those in the original challenge (challenge 0) across subsequent landscapes (Figure 2a-b). The graphs (Figure 2a refers to $K=2$ and Figure 2b to $K=5)$ clearly illustrate that when the random walk range is higher $(R W=0.05)$ the percentage of local peaks identical to those of the original landscape rapidly plummets with successive challenges, whereas when the range is lower $(R W=0.01)$, the decreasing curve approaches 0 more slowly. ${ }^{3}$ To understand the extent to which landscapes tend to differ in successive challenges, Figure 2c-d reports the percentage of local peaks that overlap in each pair of subsequent challenges in case of low and high turbulence and in case of $K=2$ (Figure 2c) and $K=5$ (Figure 2d). The graphs show similar landscape topology across challenges in less turbulent environments $(R W=0.01)$ while lower landscape correlation holds in more turbulent environments $(R W=0.05)$.

\section{[Insert Figure 2 about here]}

\subsection{Agents' Characteristics}

In our model an agent represents a founding team as a whole. The ultimate goal of each agent in the simulation is to find a profitable solution for each of the sequential challenges. The agents' overall performance in the simulation is the average of payoffs obtained for each of the challenges that she faces.

We define a search strategy as a set of decisions agents make to solve their set of challenges. For each challenge, this sequence of decisions is as follows: (1) definition of a starting point, (2) allocation of processing time, and (3) evaluation of alternatives. The first decision refers to the choice of initial string from which the agents explore each challenge. The second aspect indicates the amount of processing time that the agents use to explore each challenge in an attempt to find a profitable solution. Finally, once the agent has decided both the quantity of processing time that she intends to use and the string from which she intends to start her exploration, she will perform

\footnotetext{
${ }^{3}$ We did not report the graph of low complexity $(K=0)$ associated with a single local peak, as the percentage of distinct local peaks trivially converges to 0 after one or fewer steps.
} 
a series of evaluations of possible improvements of the initial string and perform a mutation only if it improves upon the status quo. An agent's solution for a given challenge is the string with the highest fitness found during the allocated processing time. We now describe two alternative search strategies that the agents may follow.

\subsubsection{Inexperienced start-ups}

The inexperienced start-up mirrors new ventures with relatively inexperienced founding teams. These agents will only adopt one possible search strategy for each challenge. In this directed exploration strategy, the starting point linked with the original challenge (challenge 0) will be randomly determined, and each starting point of subsequently generated landscapes will be drawn from the neighborhood of the most successful solution the agent found for prior challenges. In particular, to select a starting point for addressing each new challenge, the agent will first select the string with the highest fitness value among the solutions found in the past and then mutate each bit of this string with probability equal to 0.5. This mechanism aims to capture the role of prior experience: founders of inexperienced start-ups jointly develop some priors on how to address challenges (and so have a coarse, un-detailed map), but experiment with their policy choices (as their map does not detail the terrain of the focal challenge).

The processing time linked with directed exploration is allocated in a simple way. All agents start the simulation with the same amount of processing time $t_{0}$. The time allocated by an inexperienced start-up for generic challenge $m\left(A T_{m}\right)$ equals the overall remaining time $t_{m}$ divided by the number of remaining challenges $M-m$, where $M$ represents the total number of challenges in the simulation. The amount of time available at the end of the evaluation process for challenge $m$, which will constitute the remaining amount of time to be considered during the next challenge $\left(t_{m+1}\right)$, is equal to $t_{m}-A T_{m}$ (see Figure 1 for a summary of this process). As a result of this time allocation process, an inexperienced start-up will allocate the same amount of processing time for 
each of the challenges in the simulation. ${ }^{4}$

Given the amount of processing time allocated and the definition of the starting point, the evaluation process conducted for each directed exploration differs depending on the agents' search distance $(S D)$ and decision-making speed $(S P)$. The former parameter refers to the number of binary bits in a decision string the agent is able to flip in each evaluation. Higher levels of $S D$ represent agents able to evaluate longer jumps in the landscape space (Levinthal 1997).

The second parameter that characterizes the agents' evaluation process is team decision-making speed $(S P)$. Given the time allocated to solve a certain challenge, agents differ in the number of evaluations that they may be able to perform. We define $S P$ as the number of evaluations that she is able to perform in one unit of time. Note that the integer 1 does not represent the smallest possible unit of time. Also, if a fraction (or multiple) of the integer 1 is considered, the related number of evaluations will be rounded to the next closest integer. ${ }^{5}$ As a result, the number of evaluations performed by inexperienced start-ups attempting to improve its starting point for each challenge will be constant and equal $A T * S P$, i.e. the time allocated to the particular challenge multiplied by the speed of the agent expressed in terms of number of evaluations performed in a time unit equal to 1.

\subsubsection{Experienced new ventures}

The second type of agent encompasses either those firms that have developed a sufficient level of experience over time or start-ups whose management team members have a substantial amount of prior joint experience in founding and/or operating a new venture. ${ }^{6}$

\footnotetext{
${ }^{4}$ This mechanism implies that agents are aware of both the total number of challenges in the simulation and the amount of time they have to solve them. The former assumption is based on the premise that founding teams are generally aware of the level of organizational development necessary for a favorable outcome, such as launching an initial public offering. Founding team and/or investor-imposed deadlines for addressing challenges that may be time and/or event-based motivates the latter assumption (and is consistent with the findings of Gersick (1994)).

${ }^{5}$ Therefore, if an agent's SP equals 10, in 0.34 units of time she will perform 3 evaluations.

${ }^{6}$ While we envision different event-based goals/deadlines for inexperienced start-ups (becoming an experienced venture) and experienced ventures (becoming an established firm), from a modeling standpoint, all agents face 300
} 
The distinctive characteristic of such experienced new ventures is that they may adopt protoroutines. Experienced new ventures therefore have the choice of two strategies for addressing each of their challenges. The first option is a directed exploration as previously detailed for inexperienced start-ups. The second option is using a protoroutine. In contrast to the case of directed exploration where each new starting point was in the neighborhood of the most successful past solution, each new starting point using a protoroutine exactly coincides with the most successful solution from the stock of preceding challenges. This mechanism captures the deliberate and conscious aspect of protoroutines in the context of founding team decision-making. In addition, in accordance with the unspecific target aspect of protoroutines (as compared to established organizational routines), teams do not match the set of problems to each specific protoroutine. ${ }^{7}$ When an agent applies a protoroutine for challenge $m$, she will perform no further evaluations in an attempt to improve the initial string. The time allocated to implementing this strategy, $A T$, is equal to the low arbitrary value of $1 / S P .^{8}$

Because of its cognitive efficiency, the application of a protoroutine will only "cost" a limited amount of processing time relative to directed exploration. As a result, by using a protoroutine, the firms will be able to bank time, thereby creating a buffer to be used for future directed explorations. However, recall that applying protoroutines has a downside when compared to directed exploration. In a directed exploration, the agent adapts to the specific NK challenge by attempting to improve the starting point. In contrast, a selected protoroutine may fail to represent a profitable solution for challenge $m$ given the ever-evolving landscape topology.

The decision of implementing a protoroutine as opposed to a directed exploration for a focal

successive challenges. The exact nature of individual challenges, however, can be conceptualized flexibly.

${ }^{7}$ For the sake of simplicity we only consider past fitness levels as the basis for selecting protoroutines, although other possible selection criteria are possible. Furthermore, we tested result robustness with an exponential organizational "forgetting" curve in which memory of the most fit prior solution fades with non-use over time. We selected parameters that match the new venture development process, and the results are not significantly different than the ones reported below.

${ }^{8}$ The denominator of this expression aims to capture differences among agents' decision-making speed. Although the amount of allocated time in applying protoroutines will be generally small, faster agents will use proportionally lower processing time. 
challenge is another facet of the deliberate decision-making process that distinguishes protoroutines from well-established routines. This decision is stochastically determined over time by a monotonically non-decreasing function of the number of processed challenges, which defines the agent's confidence level. Moreover, the agent will increase its likelihood of exercising a protoroutine over time if, in its last application, the protoroutine generated more positive feedback. ${ }^{9}$ Figure 1 summarizes the search strategies available to experienced new ventures.

[Insert Figure 1 about here]

\section{Results}

We use computer simulations to compare the overall performance of different types of young organizations operating in different environments. ${ }^{10}$ In doing so, we considered the following parameter space in the simulations. For all agents, we set the number of new venture decisions $(N)$, the number of challenges each agent faces $(M)$, and the initial total time availability $\left(t_{0}\right)$, equal to 10, 300 and 300, respectively. We then set two possible ranges of the random walks to simulate environmental turbulence, $R W=\{0.01,0.05\}$ and three levels of complexity, $K=\{0,2,5\}$. With respect to the agent's characteristics, we considered nine levels of decision-making speed, $S P=\{10$, $15,20,25,30,35,40,45,50\}$ and three levels of search distance $(S D)$ equal to 1,3 , and 5 . We organize our results by first discussing the performance impact of search speed and search distance, together with the moderating role of protoroutines. We then turn our attention to the relationship between organizational contextual factors (environmental turbulence and decision complexity)

\footnotetext{
${ }^{9}$ Specifically, the confidence function equals:

$$
C_{P R}= \begin{cases}C_{m-1}+C_{m-1}\left(z+\left(s * f_{P R}\right)\right) & C<h \\ h & C \geq h\end{cases}
$$

where $m$ is an arbitrary challenge, $z$ and $s$ are two arbitrary coefficients $<1, h$ is an arbitrary boundary limit beyond which the confidence level stabilizes, and $f_{P R}$ represents the fitness obtained by protoroutines. We set $h$ to 0.8 in our simulations, but the results are robust to a range of alternative values.

${ }^{10}$ To develop the code and run the simulations we used the open source software, Laboratory for Simulation Development (http://www.labsimdev.org/Joomla_1-3/).
} 
and performance, again discussing the moderating role of protoroutines. A third section discusses the joint performance effects of agent characteristics and protoroutine development under different organizational context configurations. A final section discusses result robustness.

\subsection{Performance effects of search speed, search distance, and protoroutines}

Since founding team characteristics influence firms' search speed and search distance, we examine the relationship between these parameters and organizational performance. Afterwards, we analyze the extent to which protoroutines moderates this same relationship.

Let us first consider the relationship between team decision-making speed $(S P)$ and firm performance. A general finding, which holds for each level of complexity and turbulence, is a positive performance effect produced by $S P$. In Figures 3-5, where speed is plotted on the x-axis and performance on the y-axis, each performance curve is monotonically non-decreasing in $S P$. However, the advantage of agents performing more evaluations in a given time unit varies depending on whether the firm is an inexperienced or experienced new venture: the performance benefits of decisionmaking speed are higher for the former compared to the latter (the slopes of the derivatives in Figures 3-5 $[\mathrm{a}, \mathrm{c}]$ are systematically higher than those in the Figures 3-5 [b,d]). Consequently, time buffers generated by protoroutines may partly compensate for slower founding team decisionmaking speed. On the other hand, in the absence of protoroutines, agents' speed represents a key performance booster.

These results shed light on the debate in the literature on the effect of decision-making speed. The proposition that speed is associated with performance in turbulent environments is not controversial (e.g., Eisenhardt (1989)). However, some studies have found that speed is also beneficial in stable environments (Keck 1997; Baum and Wally 2003) while others have found that faster decision-making speed is undesirable in stable environments (Judge and Miller 1991). Our results are consistent with the former view, and may stem from the unique challenge new ventures face, 
that opportunity windows may open and shut even in relatively stable organizational environments.

Let us now consider the relationship between search distance $(S D)$ and performance. We find that the optimal level of search distance is contingent on decision complexity. Under low complexity $(K=0)$, agents with the lowest $S D$ obtain superior performance regardless of the settings of the other parameters in the simulation (Figure 3a-d), but the optimal search distance varies with moderate and high complexity. With moderate complexity $(K=2)$ the agents displaying the intermediate and high search distance tend to outperform especially as decision-making speed increases (Figure 4ad). Finally, in case of high complexity $(K=5)$, agents with higher search distance tend to generally outperform (Figure 6a-d).

Therefore, agents with higher search distance obtain superior performance in the presence of higher complexity. These findings are surprising if compared with the prior findings in the NK literature (Levinthal 1997; Rivkin and Siggelkow 2003). The fact that searching at a low distance (under low complexity) can be profitable stands in contrast to the intuitive idea that agents that explore a wider portion of the landscape have an advantage over agents that may only perform evaluations at a lower search distance. In probing this result in more detail, we found that our settings uncovered a unique property of NK fitness landscapes. The relationship between $S D$ and agents' fitness values for different levels of complexity is sensitive to the number of evaluations that the agent performs to explore the landscape, which is linked with decision-making speed. ${ }^{11}$ When $K=0$, the fitness landscape contains a single peak, and performing a few long jumps in the landscape is less profitable than searching locally, whereas with higher complexity (and rugged landscapes), the former search strategy is best, as the agent is more likely to get stuck in a local peak or realize only small performance improvements by searching the immediate neighborhood. Therefore, as opposed to the case of search speed, we did not find any strong influence of adopting

\footnotetext{
${ }^{11}$ We analyzed this property by replicating the analysis for inexperienced agents after setting the speed of the agents at a significantly higher level ( $\mathrm{SP}=500$, a level ten times higher than the highest parameter value we employ in our parameter space), thus allowing the agents to perform a high number of evaluations. The results confirm that with a higher number of evaluations, agents with the highest search distance generally outperform others.
} 
protoroutines on the optimal search distance. ${ }^{12}$

[Insert Figures 3,4,5 about here]

\subsection{Performance effects of environmental turbulence, decision complexity, and protoroutines}

Here we highlight the comparative performance of inexperienced and experienced agents at different levels of turbulence and complexity. Comparisons across the graphs in Figures 3-5 show that experienced agents tend to achieve better results as environmental turbulence decreases (compare Figures 3-5 [b] vs. [d]) and as complexity increases (compare Figures 3 [b,d] vs. Figure 4 [b,d] vs. Figure $5[\mathrm{~b}, \mathrm{~d}])$. On the other hand, inexperienced agent performance tends to be invariant to turbulence levels (compare Figures 3-5 [a] vs. [c]), but these inexperienced agents obtain higher fitness in case of moderate complexity (compare Figure 4 [a,c] vs. Figure 3 [a,c] and Figure 5 [a,c]).

Let us examine the effect of protoroutines in different turbulence settings in more detail. Higher environmental turbulence is detrimental for the performance of experienced agents whereas it does not negatively influence the performance of inexperienced agents. Consider the evolution of search strategy characteristics for experienced agents operating in low vs. high turbulence environments. The level of confidence in exercising a protoroutine is higher when the environment is less turbulent $(R W=0.01)$; accordingly, in this circumstance, protoroutines are used more frequently and therefore banked time is higher on average. However, using protoroutines in rapidly changing landscape topographies tends to produce negative fitness due to the higher mismatch between solutions and task environment characteristics. Inexperienced start-ups, on the other hand, continuously engage in solution adaptation (directed exploration) across challenges, and so performance for these agents tends to be invariant to environmental turbulence. Figure 6 shows these results more clearly by comparing the fitness values of an inexperienced with an experienced agent across challenges

\footnotetext{
${ }^{12}$ One exception is the case of intermediate complexity where, as compared with inexperienced agents, protoroutines tend to favor teams that search at higher distance and low to moderate speed.
} 
under low and high environmental turbulence $(R W=0.01$ and 0.05 , respectively). In these plots, all the other settings of the simulation are held constant across the agents (with intermediate levels of complexity, search distance, and speed). For experienced agents, the performance of both protoroutines and directed explorations degrade with turbulence, while fitness is relatively constant for the inexperienced agent (compare Figure $6[\mathrm{c}, \mathrm{d}]$ with $[\mathrm{a}, \mathrm{b}]$ ). At the same time, average fitness is higher for the experienced agent under low turbulence but higher for the inexperienced agent when the environment is highly turbulent (contrast Figure $6[a, c]$ with $[\mathrm{b}, \mathrm{d}]$ ).

With regard to decision complexity, our primary finding is that experienced agent performance increases with complexity. To see this result more clearly, Figure 7 compares the fitness values of an inexperienced with an experienced agent across challenges under varying levels of decision complexity $(K=0,2$, and 5 , respectively). In these plots, all the other settings of the simulations are held constant across the agents (with intermediate levels of search distance, speed, and turbulence). For experienced agents, the performance of protoroutines and directed explorations increase with complexity, whereas the same pattern does not hold for inexperienced agents (compare Figure 7 $[\mathrm{d}, \mathrm{e}, \mathrm{f}]$ vs. $[\mathrm{a}, \mathrm{b}, \mathrm{c}])$. These results suggest that understanding the mapping between decision choice and payoffs is particularly useful when the fitness landscape is very rugged. Protoroutines allow the organizational to access past problem solutions, which in turn may (imperfectly) help map these payoffs. Through a process by which experienced agents' protoroutines yield higher performing directed explorations and vice-versa, experienced agents build a more detailed map, which can be particularly useful under complexity.

[Insert Figures 6,7 about here] 


\subsection{Joint performance effects under different organizational context configura- tions}

In this section we build on our prior results to examine the joint performance effects of founding team characteristics (as they impact search speed and search distance) and new venture evolution (protoroutines) under different configurations of decision complexity and environmental turbulence. The logic behind this analysis is three-fold. First, as previously discussed, there is an inherent tradeoff in team design such that search distance (associated with more diverse teams) is often at odds with search speed (associated with more uniform teams). As the recent literature has suggested, designing teams that are both uniform and diverse on different dimensions may circumvent this tradeoff. However, the literature does not give guidance on when it is preferable to have some elements of both. Second, protoroutine development may be a function of business policy (as well as naturally evolving over time). It is thus important to understand when to try to spur or dampen protoroutine development. Moreover, there may be situations in which composing such teams may not be feasible. Third, examining the joint effects of the above two effects enlarges the firm strategy space because it is possible to use protoroutines to accomplish some of the same functionality enabled by founding team design (search speed), and so protoroutines may be particularly helpful when there are tradeoffs at the team design level. We therefore consider four different contextual configurations in which each of complexity and turbulence can be low or high. For each configuration, we discuss the joint founding team and venture development performance effects, which are summarized in Table 1.

[Insert Table 1 about here]

\subsubsection{Low complexity and low turbulence}

When decisions are relatively independent and the external environment is stable, teams with high search speed and low search distance are preferred. The positive impact of search speed is invariant 
to all contextual configurations, while the overall search distance effect is driven by the negative impact of high search distance (the effect of $S D$ is invariant to environmental turbulence). ${ }^{13}$ The combination of high $S P$ and low $S D$ is relatively easy to implement by designing a uniform team. Protoroutines can also add performance value, as they work well in low turbulence while not having a detrimental effect under low complexity.

\subsubsection{High complexity and low turbulence}

When decisions are highly interdependent but the external environment is stable, teams with both high search speed and distance are preferred. Search distance benefits in highly complex situations drive the overall positive $S D$ effect. Achieving both high $S P$ and $S D$ at the team configuration level involves mixing uniform and diverse team members together. Protoroutines are particularly beneficial in this contextual setting, as they work well in complex environments and when turbulence is low.

\subsubsection{Low complexity and high turbulence}

When decisions are not highly coupled at the same time that the external environment is turbulent, the highest performing teams are those with high search speed and low search distance for the same reasons discussed in section 4.3.1 above. As in that case, uniform teams are preferred. Protoroutines in this contextual configuration, however, are detrimental to organizational performance, as they are not helpful under low complexity and are harmful under high turbulence.

\subsubsection{High complexity and high turbulence}

Finally, when decisions are tightly coupled and the external organizational environment changes at a relatively fast pace, mixed uniform and diverse teams are best for the same reasons discussed

\footnotetext{
${ }^{13}$ It is worth noting that in Table 1 , although the pattern of signs associated with founding team characteristics is similar across levels of turbulence, our results suggest that their relative magnitude is somewhat different.
} 
in section 4.3.2 above. The results on the net effect of protoroutine development are ambiguous, however, as protoroutines are beneficial in complex situations but harmful when turbulence is high.

To gain more insight on the net effect of protoroutines in this environment, we investigate the performance of organizations that adopt protoroutines frequently despite high turbulence. Should the net effects on performance be positive in case of high complexity, one could conclude that (under the assumptions of the model) even in highly complex and turbulent settings, protoroutines may be beneficial. To set up this analysis we alter the protoroutine confidence function. In our analysis thus far, experienced agents adjust their confidence in exercising a protoroutine vis-àvis implementing a directed exploration depending on the feedback that they receive from the adoption of a protoroutine as described in footnote 9. We refer to these agents as "sensitive" to environmental feedback when choosing a solution strategy. When industry turbulence is high, however, experienced new ventures know that their performance tends to degrade, and so may instead decide to ignore feedback from the environment as specified in the protoroutine confidence function. As a result, these types of agents will increase their protoroutine confidence by following a monotonic function that increases solely depending on the number of challenges already processed. We refer to these agents as environmentally "insensitive."

In Figure 8, we show graphically that these agents develop higher confidence in protoroutine adoption over time, leading to a greater time buffer, all else equal. In Table 2, we report performance results obtained by insensitive vs. sensitive experienced agents under high turbulence $(R W=0.05)$ and varied levels of decision complexity. The values in the table represent relative agent performance by differencing insensitive from sensitive agent performance (and so positive values, in bold, are situations in which insensitive agents outperform sensitive ones). The comparison reveals that the fitness values associated with insensitive agents are systematically higher relative to sensitive agents in case of high complexity and lower in case of moderate complexity, whereas they tend follow a more irregular pattern in the case of low complexity. In this analysis, although the magnitude at 
which the landscape topography shifts over time is invariant for insensitive and sensitive agents, differences in the evolution of time buffers generated by agents exist due to the different confidence function in adopting protoroutines. Taken together, these results suggest that even in turbulent environments where protoroutines generally underperform, when decision complexity is high, a higher reliance on protoroutines (and the resulting time buffers generated) yields performance benefits, at least within our selected parameter space. We conclude that when complexity and turbulence are both high, it is beneficial to design teams that are both uniform and diverse, and protoroutine development may further enhance performance.

[Insert Table $2 \&$ Figure 8 about here]

\subsection{Results Robustness}

\subsubsection{Robustness to selected parameter space}

Throughout the simulations, we set the number of decisions to make $N$ equal to 10 . The remaining parameters were chosen on the basis of reasonable low, medium, and high values. Regarding the value of $K$, we observed a progressive evolution of our results as the parameter ranged between 0 and 5. We therefore chose three representative values to facilitate our discussion of results. We followed a similar approach to select search distance values. For the parameter $S P$, we included a higher range of values to mitigate the risk of capturing only a portion of the overall effects. Our speed results are robust to a wider span of values (except for much higher values of $S P$, as discussed above).

We also differentiate, as previously discussed, between stable and turbulent industries by varying the random walk range that defines the magnitude of change in the sequence of challenges. More stable industries are simulated with a $R W$ value of 0.01 ; more turbulent industries take on an $R W$ value of 0.05 . To check result sensitivity, we varied the values of $R W$. The results are similar but with lower magnitude for values of $R W$ lower than 0.05 and higher than 0.01 . The results 
become unstable for RW values beyond 0.05. Larger random walk value ranges generate strongly uncorrelated landscapes at each time step, and while we wish to model some degree of environmental turbulence, our setup is not well-equipped to deal with strongly discontinuous environments (as in Tushman and Anderson (1986)). We also tested the reliability of our operationalization of industry turbulence with a measure of environmental uncertainty that has been validated in the literature, and found consistent results. ${ }^{14}$

\subsubsection{Robustness to alternative starting landscapes}

To avoid the risks of result sensitivity to a specific pattern of random binary strings chosen in the simulation, we averaged all our fitness values across 20 different starting landscapes, for each landscape, across 20 randomly chosen agents, and for each of these 400 combinations, across 300 different challenges. Because the firms that adopted the protoroutines will tend to directly implement the configuration that in the past generated the highest performance, one could suspect that the stochastically determined differences in the evolution in the inventory of protoroutines will create a bias in the results. To address this, we created a history of each agent's initial life by running 100 time steps before starting each of the 400 different simulations of an experienced new venture that composed the averages that we use for each setting of the parameters. To each of the 400 independent runs, we attributed a random search distance $(S D)$ and a random decision-making speed $(S P)$ within the range considered in our parameter space.

\footnotetext{
${ }^{14}$ This empirical measure has been used and validated by several studies in the literature (Dess and Beard 1984; Keats and Hitt 1988; Carpenter and Fredrickson 2001). To calculate a performance indicator at the industry level, we summed all the fitness values obtained by our population of agents for each challenge in both the stable and turbulent industries. We then selected ten contiguous blocks of five subsequent challenges as inputs to predict the industry trend at the sixth challenge. These values were generated by calculating the ratio between the standard error of the predictor coefficient that was obtained by regressing the industry performance indicators over the five-year blocks against year and average industry performance over the same time period. We then averaged these values for the ten blocks of challenges considered, thus obtaining a single value for each of the two industries. The value of the indicator of the more turbulent industry was consistently higher than that of the more stable one, thus providing some external validation.
} 


\subsubsection{Robustness to alternative search styles}

We conducted a supplemental analysis to test whether the results are sensitive to our choice of search distance definition. We define an alternative $S D$ mode that aims to capture another possible way by which founding teams may conduct decision-making evaluation. We allow the agent to search only at the $S D$ distance, a setting we label peripheral search. We therefore force the agent to flip a number of binary bits equal to $S D$ to perform each evaluation. This compares to our baseline method in which the agent may change a number of decisions lower or equal to $S D$ to perform a single evaluation. This second interpretation, labeled radial search, is similar to that used by prior studies in the NK literature (Rivkin and Siggelkow 2003). All our results hold also in case of peripheral search. However, it is worth noting that under peripheral search, the optimal search distance is much more sensitive to the agents' decision-making speed and environmental turbulence as compared to radial search, in which higher search distance tends to outperform at higher complexity regardless of the settings of the other parameters. The positive effect generated by radial search as compared to peripheral search for agents searching at higher distances is not surprising. First, note that for minimal search distance $(S D=1)$, peripheral and radial search result in identical search processes by definition. In case of higher search distances $(S D=3,5)$ radial search is more profitable than peripheral search since the former search mode holds the option of exploring both the periphery and the sub-area within it as compared to peripheral search.

Analyzing possible alternative search styles represents an interesting extension of the model. Different search styles may capture alternative ways by which founding teams integrate the individual knowledge of their members. In the cases discussed above, peripheral search may represent a team decision-making search process exploring only the periphery of members' collective knowledge whereas radial search may refer to the case in which the team uses its members' knowledge sets as a collection of resources, the integration of which may be optionally exploited to explore more distant solutions. 


\section{Discussion}

We address the research question: What is the effect of emergent organizational routines on venture performance given different founding team and environmental characteristics? We do so by first identifying distinctive traits that characterize protoroutines, organizational routines in a prototypical state. We embed protoroutines in an agent-based simulation model to examine how and when protoroutines contribute to firm performance. While relatively uniform founding teams tend to accomplish decision-making faster, more diverse teams are able to consider more distant solutions. Protoroutines enlarge the organizational strategy space because it is possible to use protoroutines to accomplish some of the same functionality enabled by founding team design (search speed), and so they may be particularly helpful when there are tradeoffs at the team design level. Using this perspective, our simulation results allow us to examine under what configuration of organizational contexts (environment turbulence and decision complexity) protoroutines are desirable. At a higher level, our approach adds to our understanding of the organizational processes in young enterprises that develop by both business policy and experience, together with their performance implications.

The existing literature on organizational routines contains little depth on the process of new routine formation, aside from the general notion that experimentation is likely necessary (Nelson and Winter 1982). We start from the premise that organizational routines are neither beneficial nor detrimental per se, as the prior literature has examined both their positive attributes such as cognitive efficiency (March 1958; Simon 1981) as well as their negative ones, such as organizational inertia (Hannan and Freeman 1988) and competency traps (March 1991). In our model, protoroutines are beneficial for experienced agents because they economize on execution time, though they are not optimized for solving focal challenges.

Our results suggest that protoroutines are beneficial at lower levels of environmental turbulence and higher levels of complexity. Like standard operating procedures, organizational routines benefit from recurrent application in contexts with little causal ambiguity. The turbulence results are 
consistent with this notion, as routinization is difficult in tumultuous conditions. Interestingly, we found that while routines should most easily develop in less complex situations, they are most profitable under high decision complexity, suggesting that it is in such contexts that entrepreneurial ventures may wish to encourage protoroutine development via business policy. Even under turbulent conditions, which tend to dampen the performance of protoroutines, we find the intriguing result that under high complexity, experienced agents ignoring performance feedback with respect to their policy choices in their protoroutine confidence function outperform similar agents internalizing such feedback. This suggests that on balance, protoroutines may be beneficial when the organizational environment is both complex and turbulent. Indeed, of the four joint organizational environmental configurations we consider, protoroutines contribute to firm performance in all cases except under low complexity and high turbulence.

We also contribute to the literature on founding team composition. Given the tendency of individuals to associate with common others, founding teams are more homophilous relative to what would be expected from the distribution of potential founding teams (Ruef et al. 2004). These more homogenous teams tend to excel at decision-making speed but lag in search distance (and vice-versa for heterogeneous teams). While the recent literature on teams suggests forming teams that are uniform in some dimensions while diverse on others, our method allows us to map ideal team composition to different configurations of organizational context (environmental turbulence and decision complexity). In that regard, our analysis informs start-up designers of the circumstances under which they may wish to "go against the grain" and add more diversity to their founding teams (our results suggest in high complexity situations). In a further contrast to the extant literature, our simulation results suggest that purely diverse teams (without at least some dimension of uniformity) are never optimal.

Our final contribution is at the nexus of team composition and protoroutine development. Since protoroutine use can accomplish some of the same functionality (decision-making speed) as 
founding team design, protoroutines add an organizational instrument which may be particularly useful when there is a search speed and search distance tradeoff at the team design level (as is the case when high complexity is paired with low turbulence, and when high complexity occurs at the same time as high turbulence).

In future work, we hope to enrich the model presented here. While our definition of environmental turbulence takes into account the influence of rapidly changing environments, we have little understanding on the possible effects of more punctuated change, which may take place even in relatively stable industries (Tushman and Anderson 1986). Moreover, in our simulations we consider the team composition as fixed. It may be interesting to evaluate the impact of changes in founding team composition on new venture performance at different points of the enterprise development lifecycle.

In conclusion, by embedding protoroutines into a model relating founder characteristics to venture performance, we gain insight into the role of time as a potentially valuable organizational resource. We believe that the resulting framework deepens our understanding of how and with what performance consequence new ventures develop. 


\section{References}

Ancona D. G., Caldwell D. F. Demography and Design: Predictors of New Product Team Performance. Organization Science 1992; 3 (3): 321-341.

Bantel K. A., Jackson S. E. Top Management and Innovations in Banking: Does the Composition of the Top Team Make a Difference. Strategic Management Journal 1989; 10 (1): 107-124.

Baron J. N., Burton M. D., Hannan M. T. The Road Taken: Origins and Evolution of Employment Systems in Emerging Companies. Industrial and Corporate Change 1996; 5 (2): 239.

Baum J. R., Wally S. Strategic Decision Speed and Firm Performance. Strategic Management Journal 2003; 24 (11): 1107-1129.

Beckman C. M. The Influence of Founding Team Company Affiliations on Firm Behavior. Academy of Management Journal 2006; 49 (4): 741.

Boeker W. Strategic Change: The Effects of Founding and History. Academy of Management Journal 1989: 489-515.

Bresnahan T. F., Brynjolfsson E., Hitt L. M. Information Technology, Workplace Organization, and the Demand for Skilled Labor: Firm-Level Evidence. Quarterly Journal of Economics 2002; 117 (1): 339-376.

Carpenter M. A., Fredrickson J. W. Top Management Teams, Global Strategic Posture, and the Moderating Role of Uncertainty. Academy of Management Journal 2001; 44 (3): 533-546.

Davis J. P., Eisenhardt K. M., Bingham C. B. Developing Theory Through Simulation Methods. Academy of Management Review 2007; 32 (2): 480-499.

Dess G. G., Beard D. W. Dimensions of Organizational Task Environments. Administrative Science Quarterly 1984; 29 (1): 52-73.

Eisenhardt K. M. Making Fast Strategic Decisions in High-Velocity Environments. Academy of Management Journal 1989; 32 (3): 543-576.

Eisenhardt K. M., Schoonhoven C. B. Organizational Growth: Linking Founding Team, Strat- 
egy, Environment, and Growth among US Semiconductor Ventures, 1978-1988. Administrative Science Quarterly 1990; 35 (3): 504-529.

Feldman M. S., Pentland B. T. Reconceptualizing Organizational Routines as a Source of Flexibility and Change. Administrative Science Quarterly 2003; 48 (1): 94-121.

Geletkanycz M. A., Hambrick D. C. The External Ties of Top Executives: Implications for Strategic Choice and Performance. Administrative Science Quarterly 1997; 42 (4): 654-681.

Gersick C. J. G. Pacing Strategic Change: The Case of a New Venture. Academy of Management Journal 1994; 37 (1): 9-45.

Gersick C. J. G., Hackman J. R. Habitual Routines in Task-Performing Groups. Organizational behavior and human decision processes 1990; 47 (1): 65-97.

Hannan M. T., Freeman J. Structural Inertia and Organizational Change. Readings in Organizational Decline: Frameworks, Research, and Prescriptions 1988.

Hong L., Page S. E. Groups of Diverse Problem Solvers Can Outperform Groups of High-Ability Problem Solvers. Proceedings of the National Academy of Sciences 2004; 101 (46): 16385-16389.

Ichniowski C., Shaw K., Prennushi G. The Effects of Human Resource Management Practices on Productivity: A Study of Steel Finishing Lines. The American Economic Review 1997; 87 (3): 291-313.

Judge W. Q., Miller A. Antecedents and Outcomes of Decision Speed in Different Environmental Contexts. Academy of Management Journal 1991; 34 (2): 449-463.

Kaplan S. N., Stromberg P. Financial Contracting Theory Meets the Real World: An Empirical Analysis of Venture Capital Contracts. The Review of Economic Studies 2003; 70 (2): 281-315.

Kauffman S. A. The Origins of Order: Self-organization and Selection in Evolution. New York, USA: Oxford University Press, 1993.

Keats B. W., Hitt M. A. A Causal Model of Linkages among Environmental Dimensions, Macro Organizational Characteristics, and Performance. Academy of Management Journal 1988; 31 (3): 
$570-598$.

Keck S. L. Top Management Team Structure: Differential Effects by Environmental Context. Organization Science 1997; 8 (2): 143-156.

Levinthal D. A. Adaptation on Rugged Landscapes. Management science 1997; 43 (7): 934950.

March J. G. Exploration and Exploitation in Organizational Learning. Organization Science 1991; 2 (1): 71-87.

March J. G., Simon, H. A. Organizations. New York: Wiley, 1958.

Murray A. I. Top Management Group Heterogeneity and Firm Performance. Strategic Management Journal 1989; 10 (1): 125-141.

Nelson R. R., Winter S. G. An Evolutionary Theory of Economic Change. Cambridge, MA: Belknap, 1982.

Paoli M., Prencipe A. Memory of the Organisation and Memories within the Organisation. Journal of Management and Governance 2003; 7 (2): 145-162.

Rivkin J. W., Siggelkow N. Balancing Search and Stability: Interdependencies Among Elements of Organizational Design. Management Science 2003; 49 (3): 290-311.

Ruef M., Aldrich H. E., Carter N. M. The Structure of Founding Teams: Homophily, Strong Ties, and Isolation among US Entrepreneurs. American Sociological Review 2004; 69 (2): 317-317.

Schweiger D. M., Sandberg W. R., Ragan J. W. Group Approaches for Improving Strategic Decision Making: A Comparative Analysis of Dialectical Inquiry, Devil's Advocacy, and Consensus. Academy of Management Journal 1986; 29 (1): 51-71.

Simon H. A. The Architecture of Complexity. Proceedings of the American Philosophical Society 1962; 106 (6): 467-482.

Simon H. A. Cognitive Science: The Newest Science of the Artificial. Cognitive Science 1981; $4(1): 33-46$. 
Smith K. G., Smith K. A., Olian J. D., Sims Jr H. P., O’Bannon D. P., Scully J. A. Top Management Team Demography and Process: The Role of Social Integration and Communication. Administrative Science Quarterly 1994; 39 (3).

Stinchcombe A. L. Organizations and Social Structure. Handbook of Organizations 1965: 142-193.

Tushman M. L., Anderson P. Technological Discontinuities and Organizational Environments. Administrative science quarterly 1986: 439-465.

Virany B., Tushman M. L., Romanelli E. Executive Succession and Organization Outcomes in Turbulent Environments: An Organization Learning Approach. Organization Science 1992; 3 (1): $72-91$. 


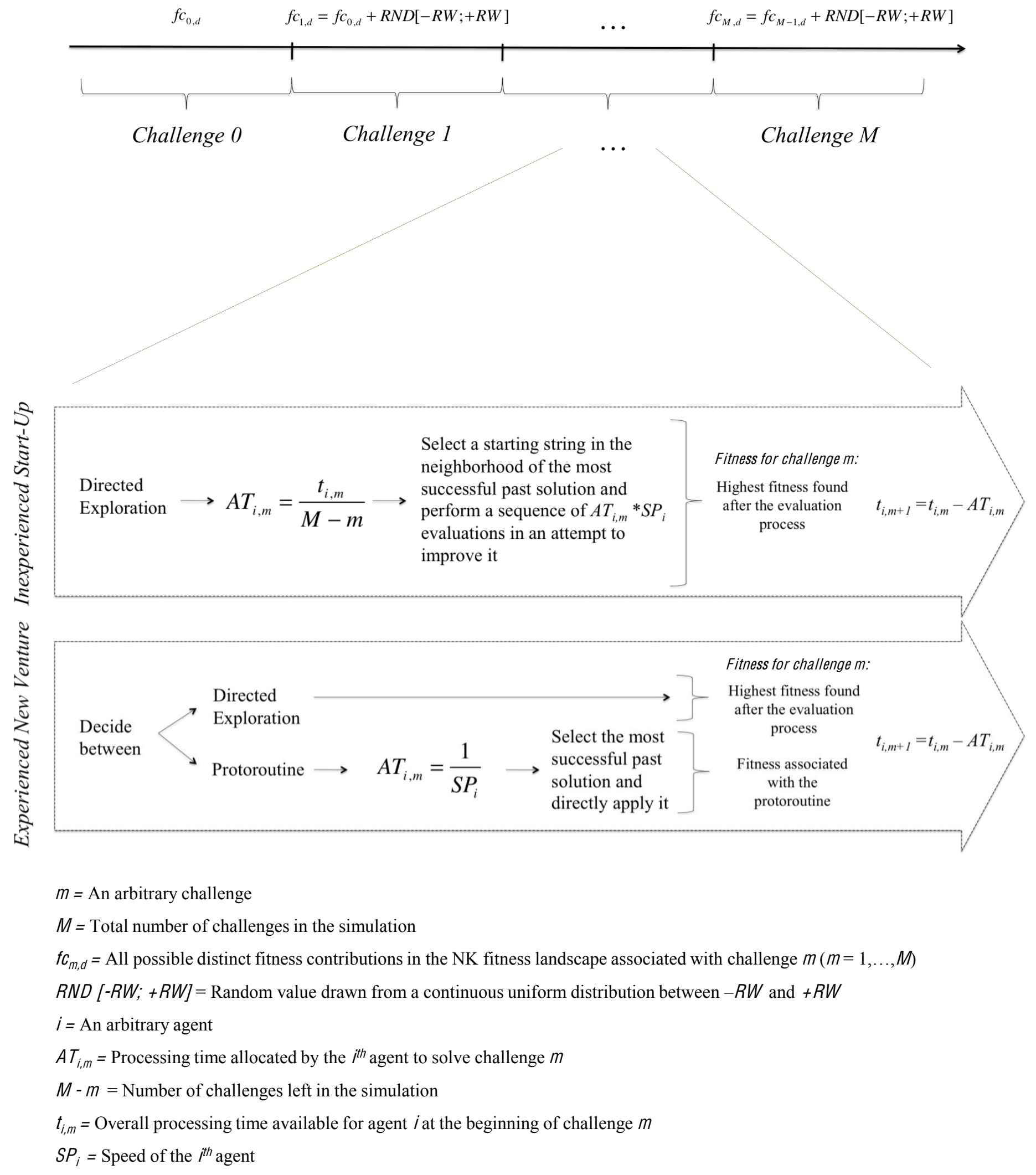

Figure 1: Description of the task environment and search strategies adopted by inexperienced start-ups and experienced new ventures 
(a) $\mathrm{K}=2$

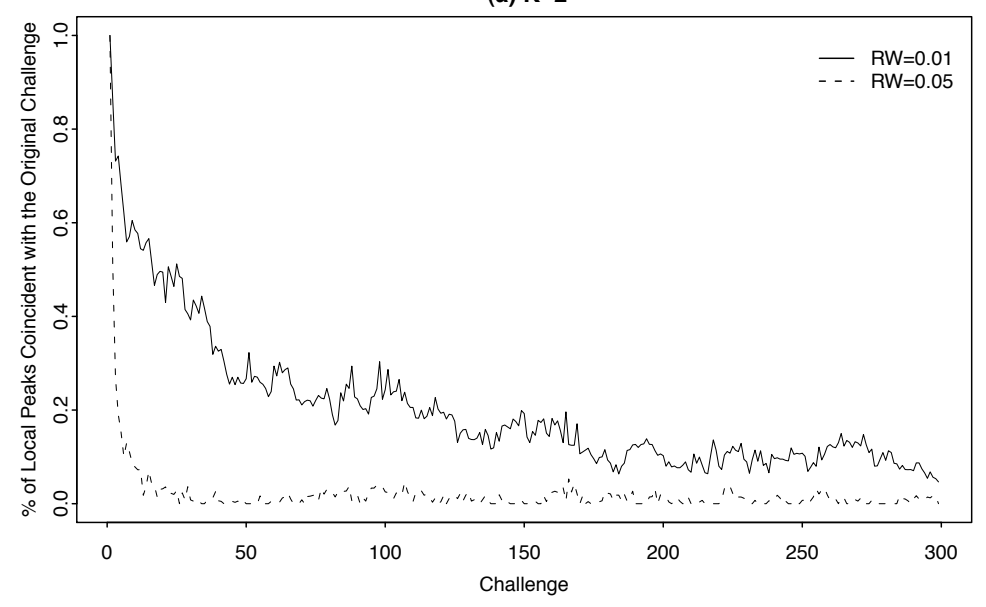

(c) $\mathrm{K}=2$
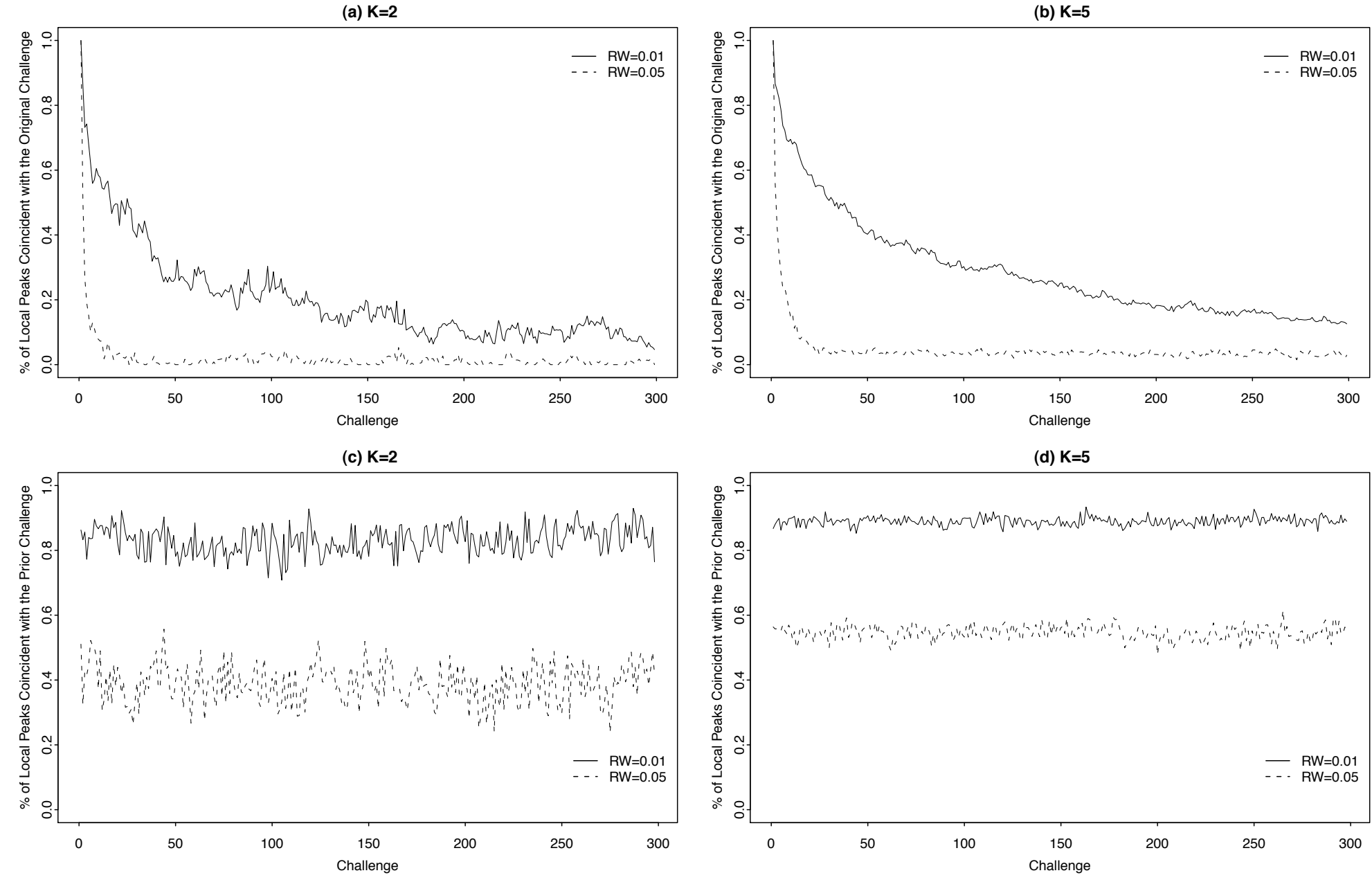

Figure 2: Description of the evolving landscape topography

Averages over 20 independent agents starting from 20 randomly generated landscapes and 300 challenges. The percentage of local peaks coincident with those observed in the original challenge (Challenge 0) is plotted on the $y$-axis. $K$ represents the level of complexity of the landscape. $R W$ represents the range of the random walk by which the fitness contributions linked with the challenges are modified over time. 
(a) Inexperienced, $R W=0.01$

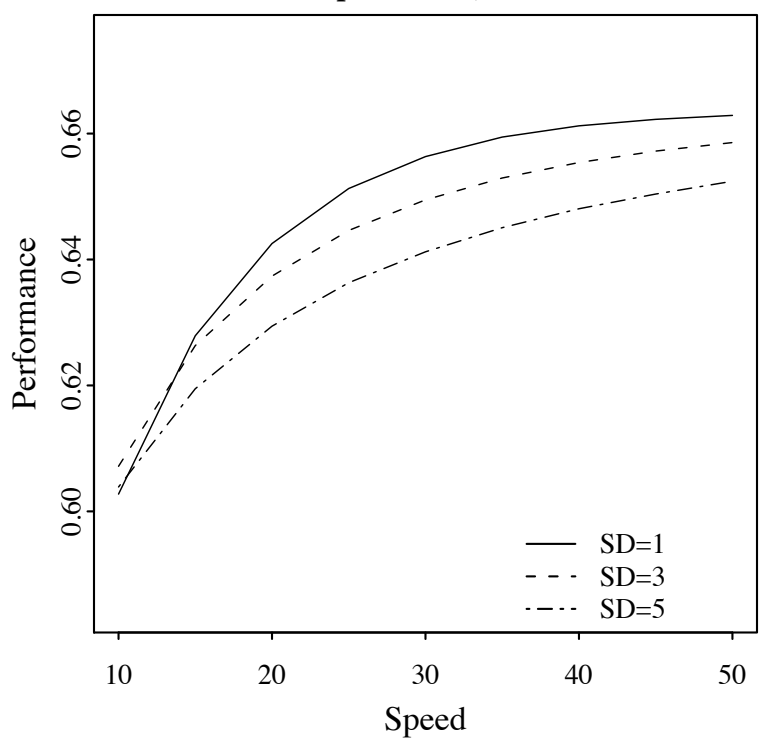

(c) Inexperienced, $R W=0.05$

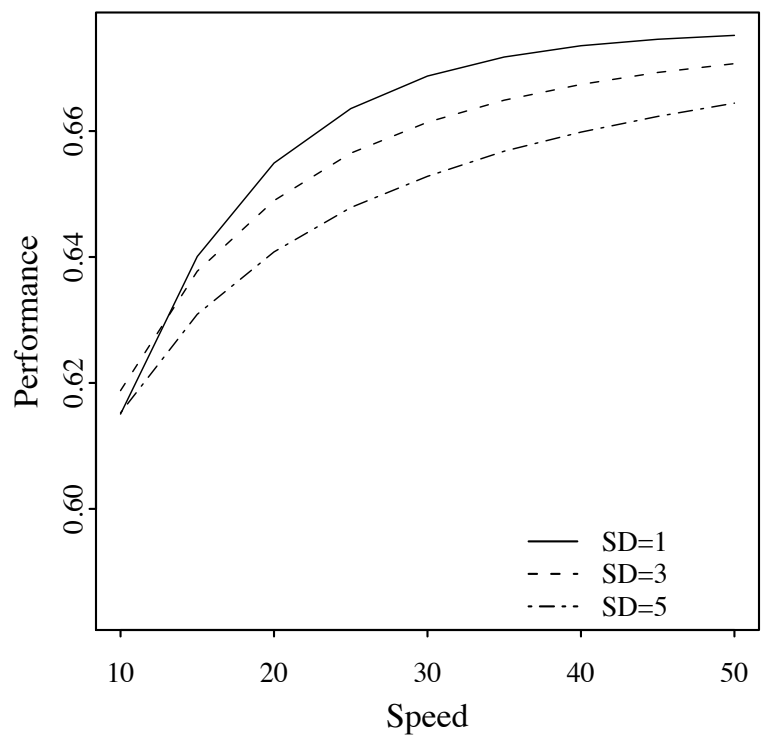

(b) Experienced, $R W=0.01$

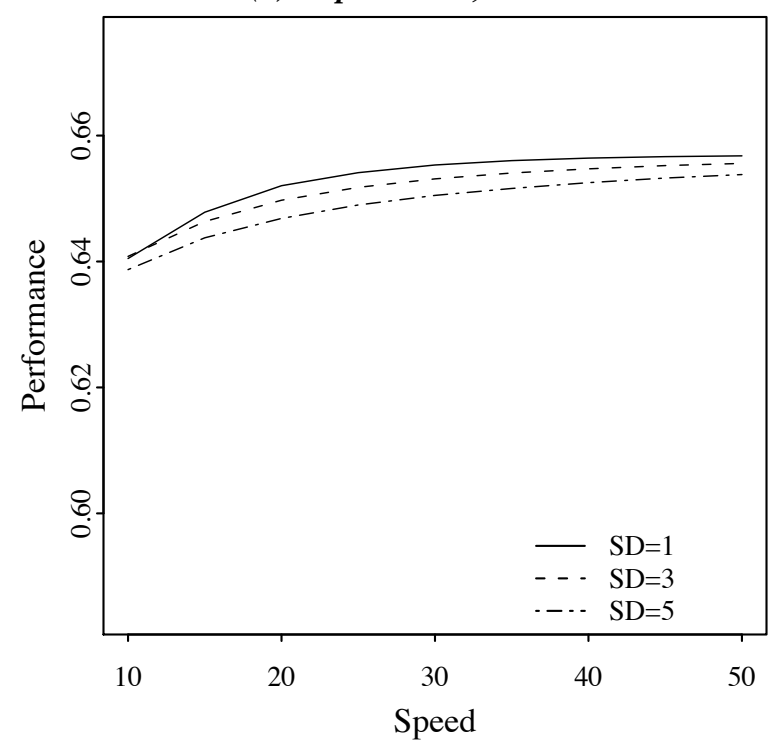

(d) Experienced, $R W=0.05$

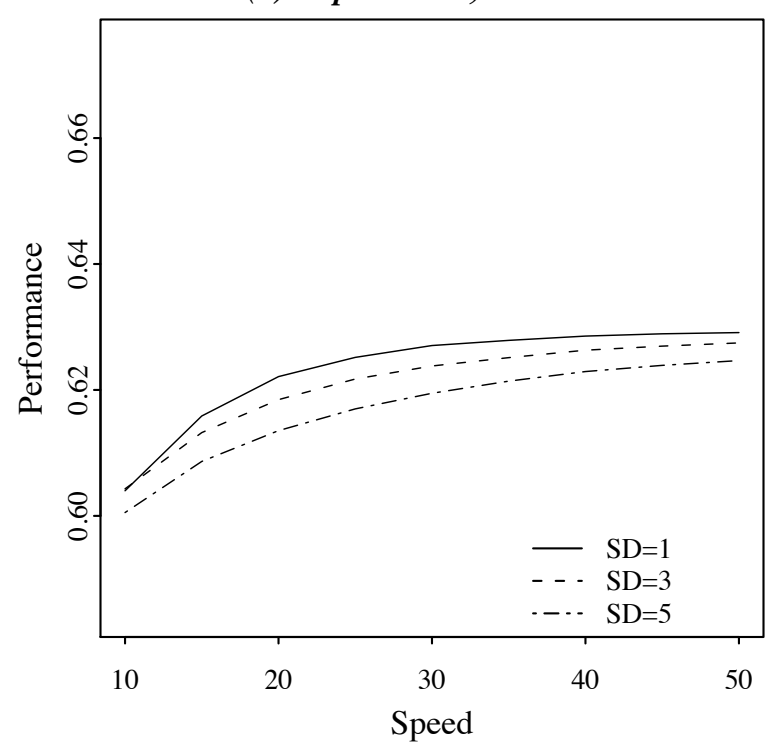

Figure 3: Comparison of the results obtained by inexperienced start-ups and experienced new ventures under low complexity $(\mathrm{K}=0)$

Averages over 20 independent agents starting from 20 randomly generated landscapes. Agent decisionmaking speed (SP) (x-axis) is plotted against average performance (y-axis). SD represents agents search distance. $K$ represents the level of complexity of the landscape. $R W$ represents the range of the random walk by which the fitness contributions linked with the challenges are modified over time. Inexperienced start-ups only adopt directed explorations; experienced new ventures adopt either directed explorations or protoroutines. For each agent: $N=10, t_{0}=300, M=300$. 
(a) Inexperienced, $R W=0.01$

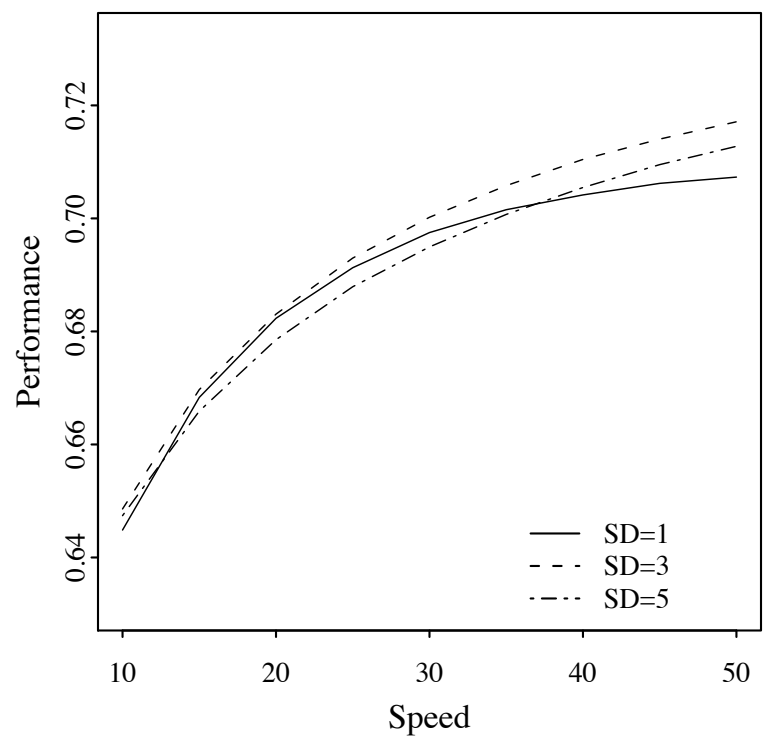

(c) Inexperienced, $R W=0.05$

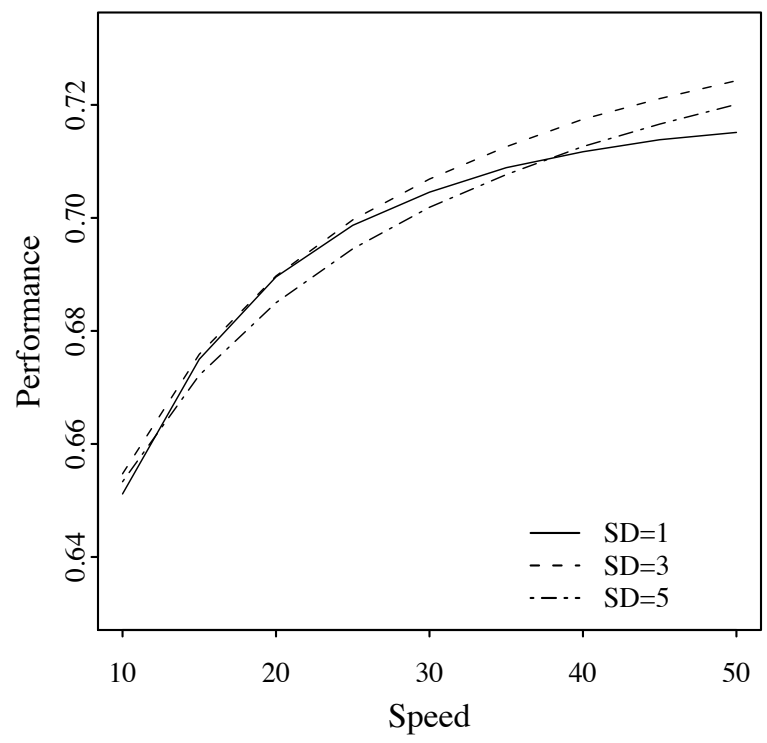

(b) Experienced, $R W=0.01$

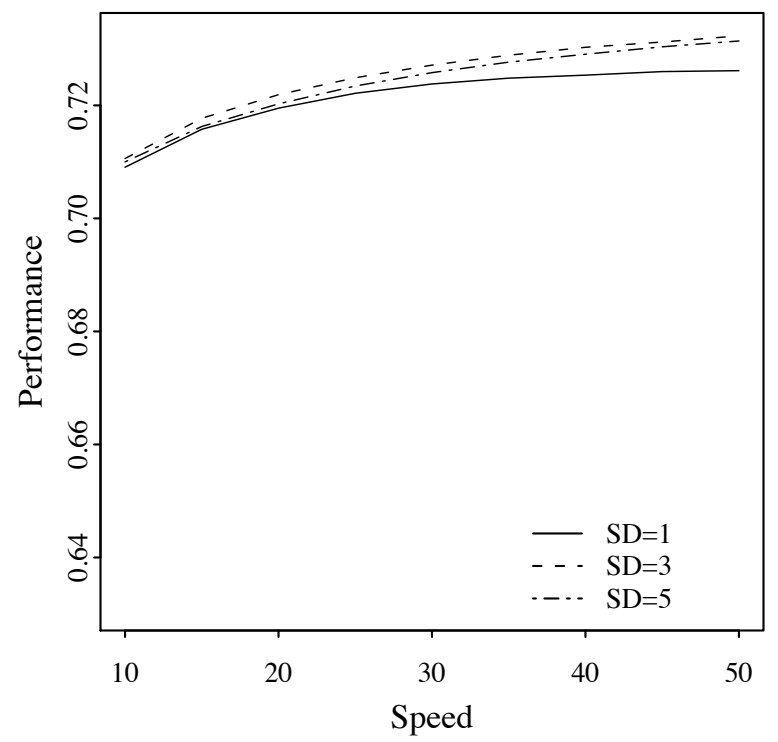

(d) Experienced, $R W=0.05$

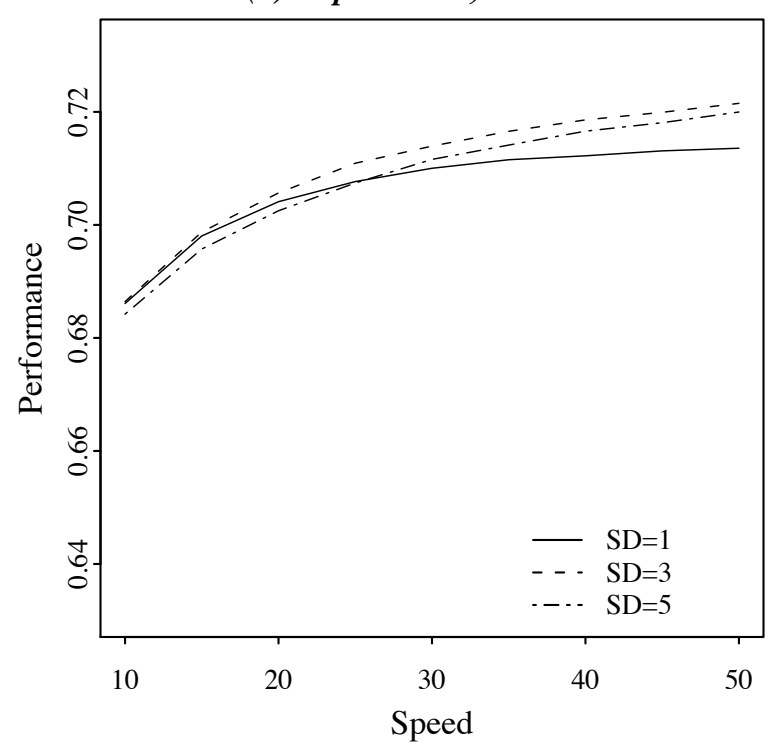

Figure 4: Comparison of the results obtained by inexperienced start-ups and experienced new ventures under medium complexity $(\mathrm{K}=2)$

Averages over 20 independent agents starting from 20 randomly generated landscapes. Agent decisionmaking speed (SP) (x-axis) is plotted against average performance (y-axis). SD represents agents search distance. $K$ represents the level of complexity of the landscape. $R W$ represents the range of the random walk by which the fitness contributions linked with the challenges are modified over time. Inexperienced start-ups only adopt directed explorations; experienced new ventures adopt either directed explorations or protoroutines. For each agent: $N=10, t_{0}=300, M=300$. 
(a) Inexperienced, $R W=0.01$

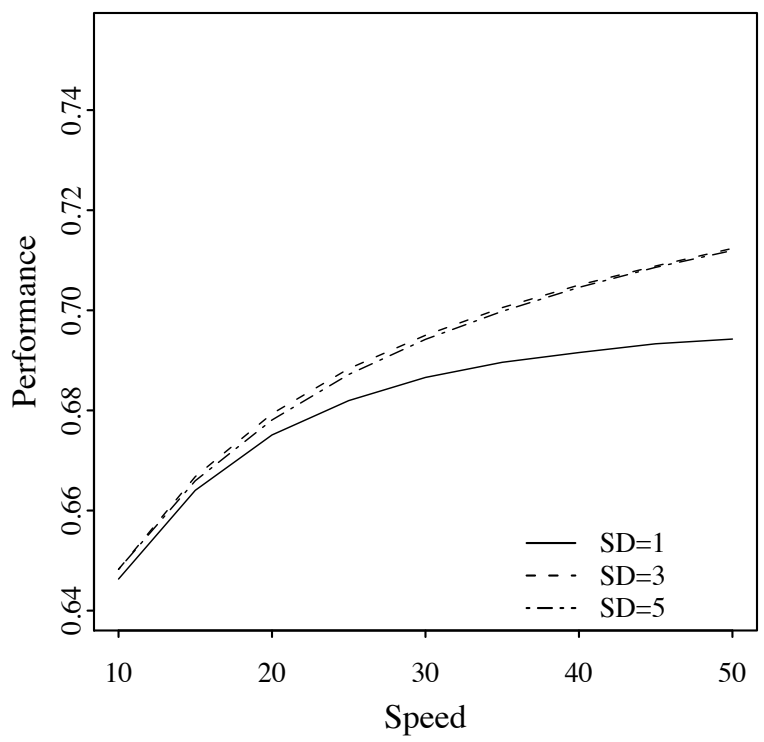

(c) Inexperienced, $R W=0.05$

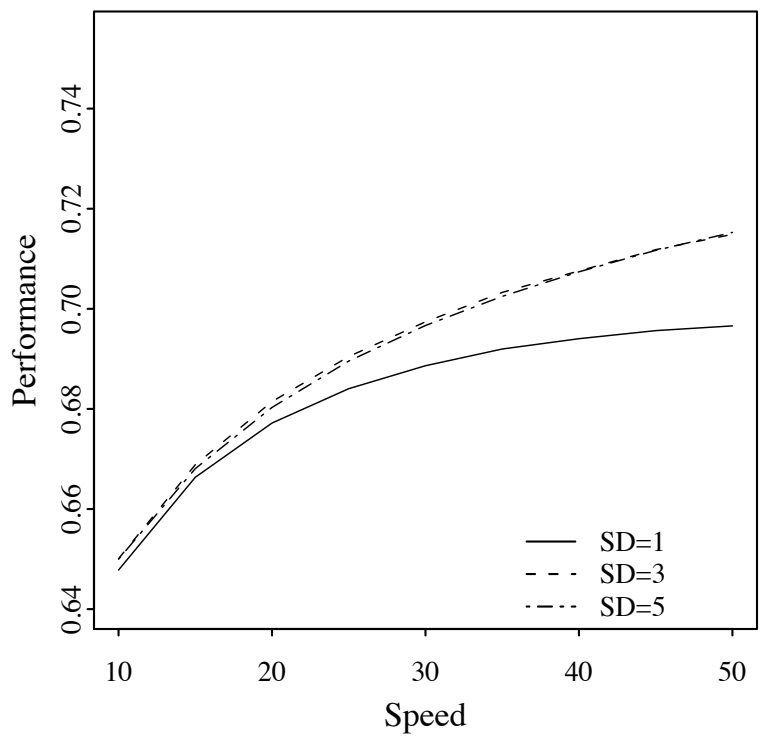

(b) Experienced, $R W=0.01$

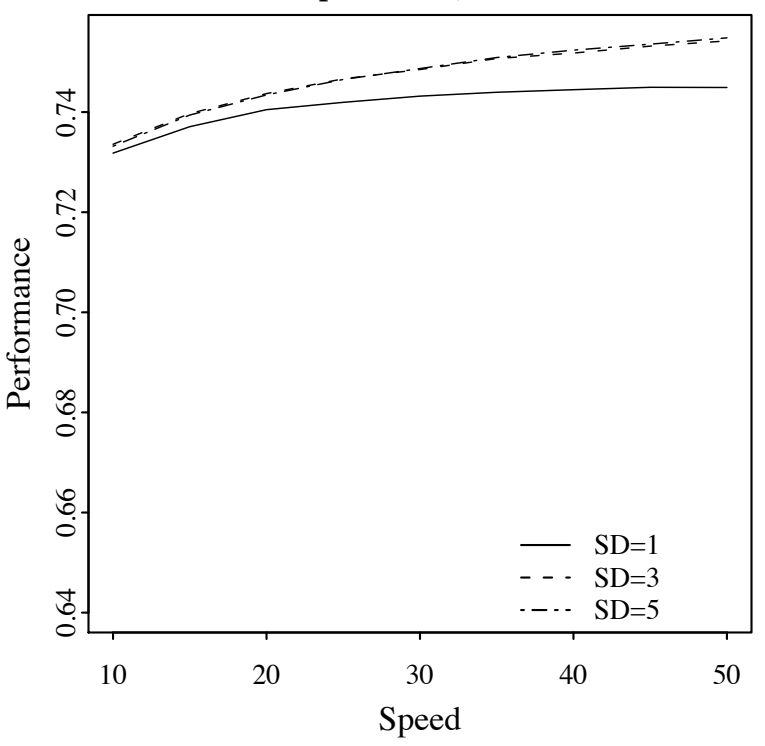

(d) Experienced, $R W=0.05$

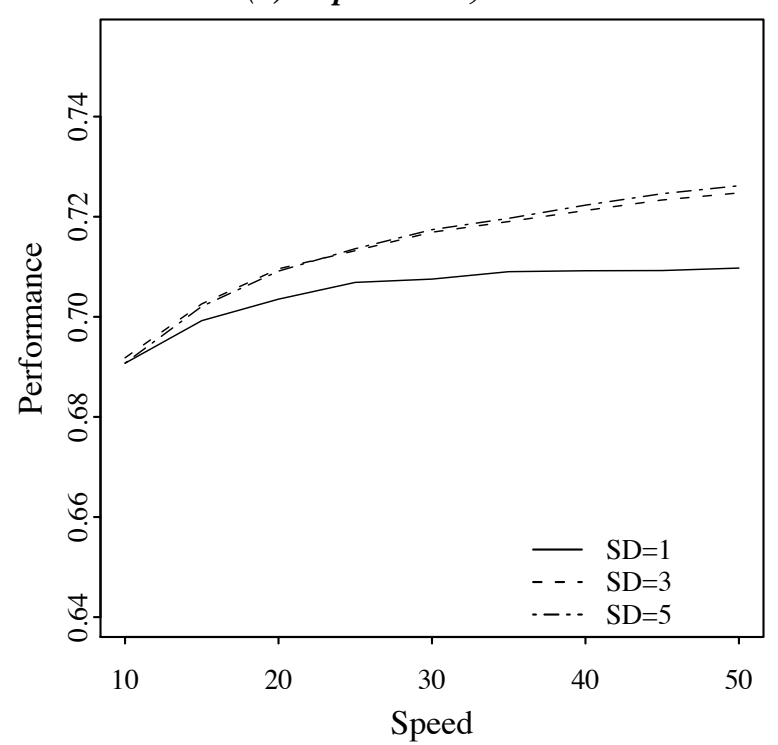

Figure 5: Comparison of the results obtained by inexperienced start-ups and experienced new ventures under high complexity $(\mathrm{K}=5)$

Averages over 20 independent agents starting from 20 randomly generated landscapes. Agent decisionmaking speed (SP) (x-axis) is plotted against average performance (y-axis). SD represents agents search distance. $K$ represents the level of complexity of the landscape. $R W$ represents the range of the random walk by which the fitness contributions linked with the challenges are modified over time. Inexperienced start-ups only adopt directed explorations; experienced new ventures adopt either directed explorations or protoroutines. For each agent: $N=10, t_{0}=300, M=300$. 

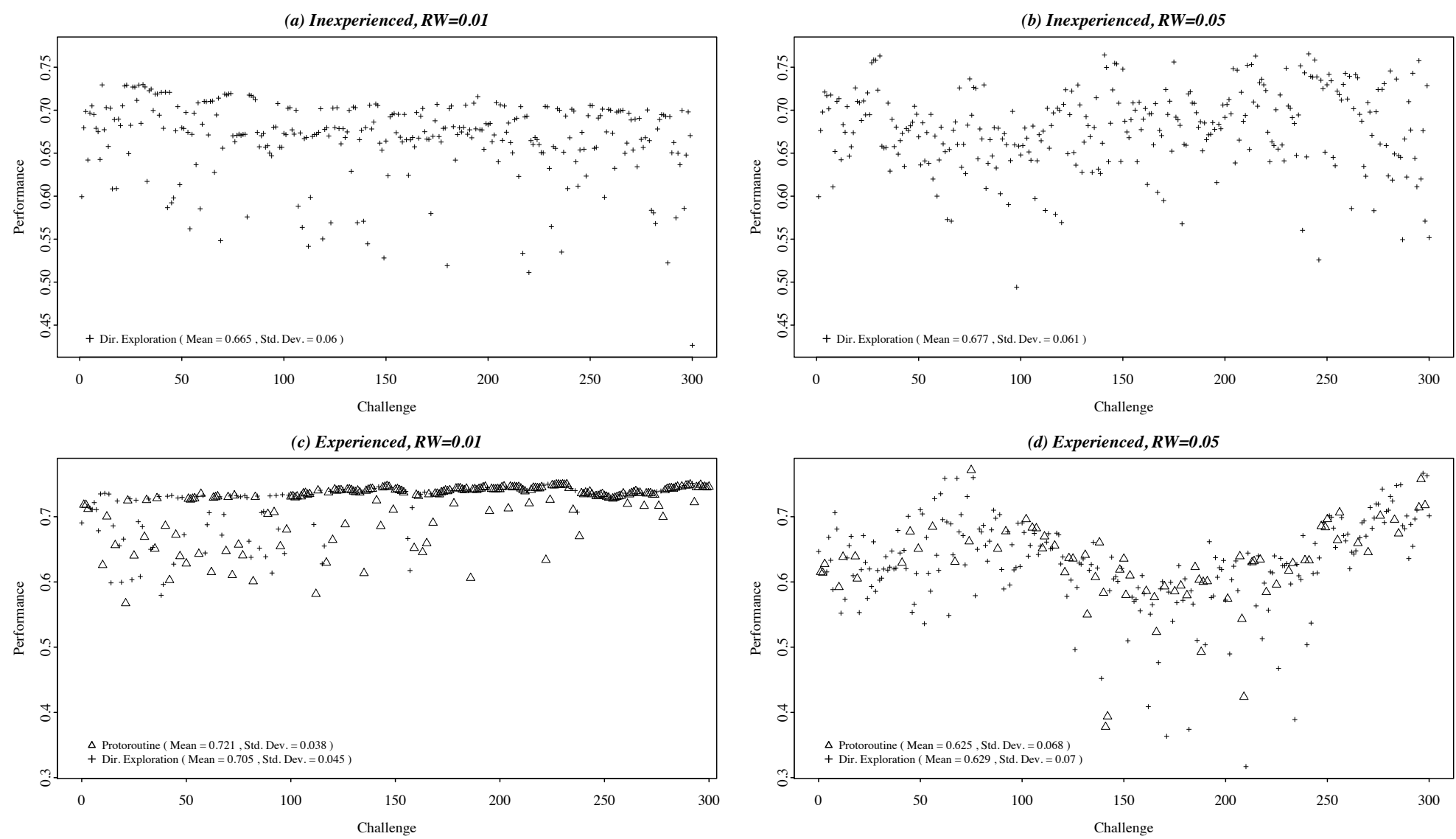

Figure 6: Comparison between fitness values generated by directed exploration and protoroutines under varied turbulence

Data generated by an individual agent operating in an arbitrary landscape. $R W$ represents the range of the random walk by which the fitness contributions linked with the challenges are modified over time. The agent is set either as an inexperienced start-up $(a, b)$ or as an experienced new venture $(c, d)$ searching peripherally with $K=2, N=10, t_{0}=300$, $M=300, S D=3$, and $S P=30$. 

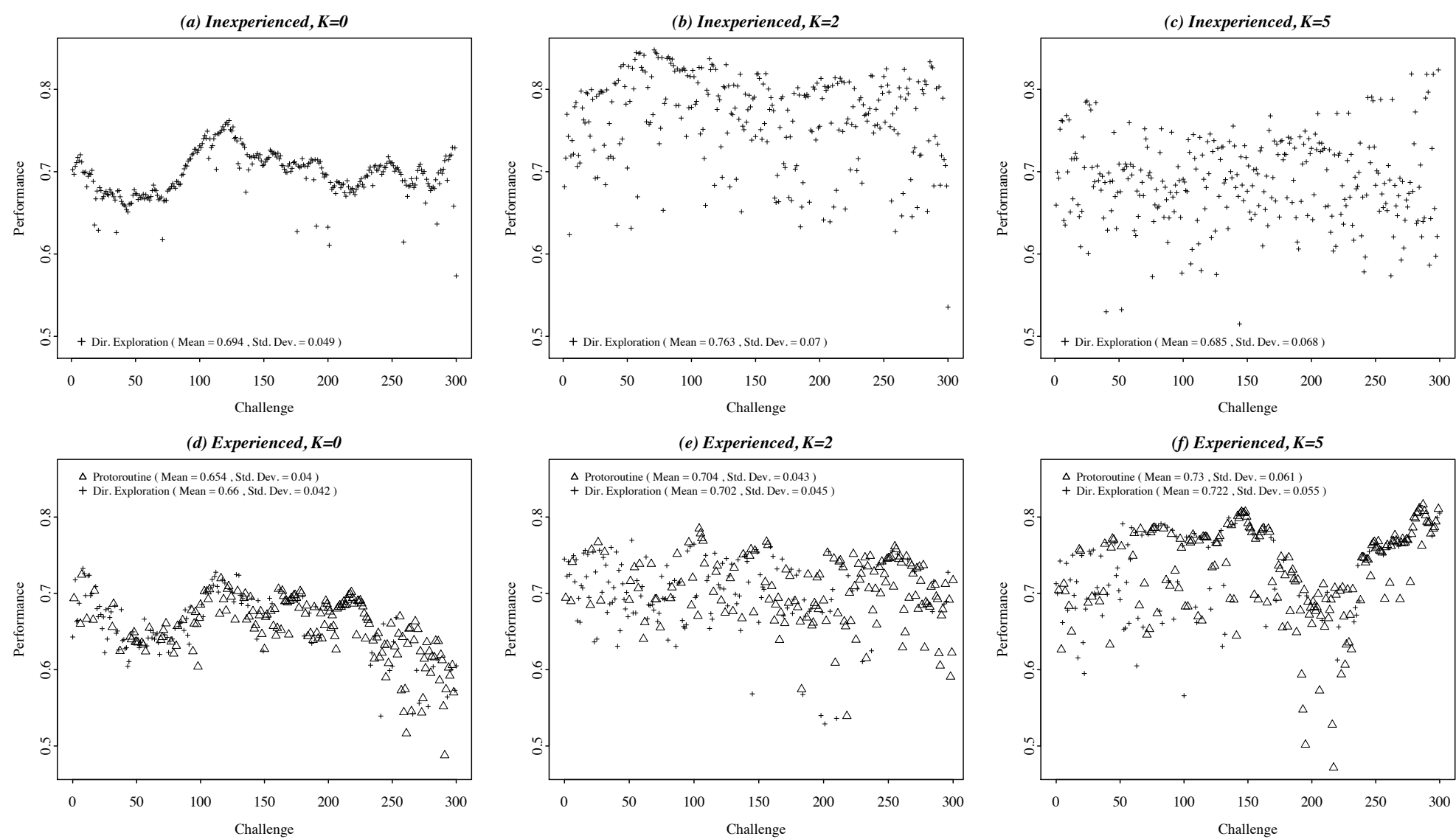

Figure 7: Comparison between fitness values generated by directed exploration and protoroutines under varied complexity

Data generated by an individual agent operating in an arbitrary landscape. $K$ represents the complexity of the setting. The agent is set either as an inexperienced start-up or as an experienced new venture with $N=10, t_{0}=300, M=300$, $S D=3, S P=30$, and $R W=0.03$. 

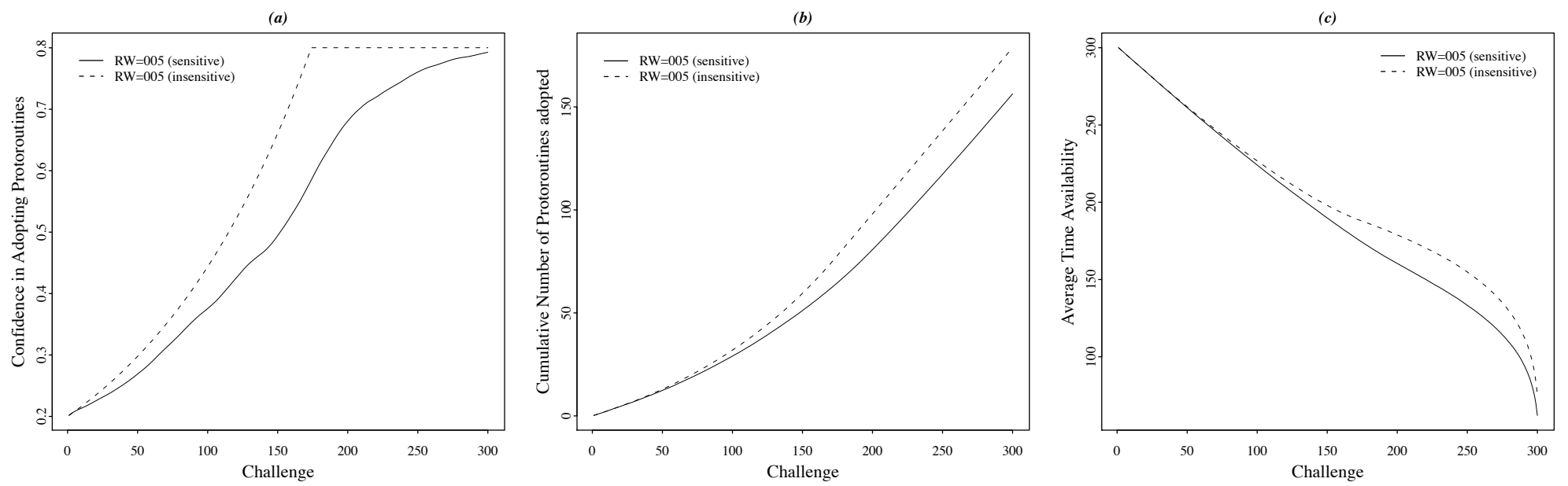

Figure 8: Comparison of experienced new ventures displaying sensitivity and insensitivity to environmental feedback in using protoroutines

Averages over 20 independent agents starting from 20 randomly generated landscapes. $R W$ represents the range of the random walk by which the fitness contributions linked with challenges are modified over time. Experienced new ventures adopt either directed explorations or protoroutines. Insensitive agents do not adjust their confidence function in adopting protoroutines depending upon the observed profitability of the protoroutines (whereas sensitive ones do adjust). For each agent: $N=10, t_{0}=300, M=300, K=2, S D=3$, and $S P=30$. 
Table 1: Comparison between fitness values generated by directed exploration and protoroutines under varied complexity

\begin{tabular}{|c|c|c|c|c|c|c|c|c|c|c|}
\hline & & \multicolumn{5}{|c|}{ Founding Team Characteristics } & \multicolumn{3}{c|}{ New Venture Evolution } \\
\hline & & \multicolumn{3}{|c|}{ Search Speed } & \multicolumn{3}{|c|}{ Search Distance } & \multicolumn{3}{c|}{ Protoroutines } \\
\hline $\begin{array}{c}\text { (a) } \\
\text { complexity }\end{array}$ & $\begin{array}{c}\text { (b) } \\
\text { turbulence }\end{array}$ & $\mathbf{( a )}$ & $\mathbf{( b )}$ & $\mathbf{( a ) + ( b )}$ & $\mathbf{( a )}$ & $\mathbf{( b )}$ & $\mathbf{( a ) + ( b )}$ & $\mathbf{( a )}$ & $\mathbf{( b )}$ & $\mathbf{( a ) + ( b )}$ \\
\hline Low & Low & + & + & + & - & 0 & - & 0 & + & + \\
\hline High & Low & + & + & + & + & 0 & + & + & + & + \\
\hline Low & High & + & + & + & - & 0 & - & 0 & - & - \\
\hline High & High & + & + & + & + & 0 & + & + & - & $?$ \\
\hline
\end{tabular}

Averages over 20 independent agents starting from 20 randomly generated landscapes. Low and high complexity equal to $K=0$ and 5 respectively whereas low and high turbulence equal to $R W=0.01$ and 0.05 , respectively. For all agents $N=10, t_{0}=300, M=300$. The columns (a) report the sign of the impact of high levels of the parameter with respect to the level of complexity indicated in the column labeled "complexity. "The columns (b) report the sign of the impact of high levels of the parameter with respect to the level of turbulence indicated in the column labeled "turbulence. "The columns (a)+(b) report the expected sign of the net impact of high levels of the parameter given the related signs of columns (a) and (b). The sign "+ " ("- ") indicates that high (low) levels of the parameters are desired, and " 0 " indicates an irrelevant impact. 
Table 2: Comparison of experienced new ventures displaying sensitivity and insensitivity to environmental feedback in using protoroutines

\begin{tabular}{|c|c|c|c|c|c|c|c|c|c|}
\hline & \multicolumn{3}{|c|}{$K=0$} & \multicolumn{3}{|c|}{$K=2$} & \multicolumn{3}{|c|}{$K=5$} \\
\hline $\begin{array}{l}\text { SD } \\
\text { SP }\end{array}$ & 1 & 3 & 5 & 1 & 3 & 5 & 1 & 3 & 5 \\
\hline 10 & 0.0062 & 0.0049 & 0.0052 & -0.0074 & -0.0073 & -0.0089 & 0.0064 & 0.0061 & 0.0058 \\
\hline 15 & 0.0024 & 0.0025 & 0.0034 & -0.0107 & -0.0105 & -0.0109 & 0.0057 & 0.0048 & 0.0049 \\
\hline 20 & 0.0002 & 0.0010 & 0.0025 & -0.0123 & -0.0122 & -0.0119 & 0.0057 & 0.0048 & 0.0039 \\
\hline 25 & -0.0009 & 0.0000 & 0.0014 & -0.0131 & -0.0139 & -0.0133 & 0.0047 & 0.0053 & 0.0047 \\
\hline 30 & -0.0016 & -0.0007 & 0.0004 & -0.0147 & -0.0148 & -0.0144 & 0.0056 & 0.0043 & 0.0044 \\
\hline 35 & -0.0018 & -0.0012 & 0.0000 & -0.0147 & -0.0154 & -0.0149 & 0.0043 & 0.0054 & 0.0043 \\
\hline 40 & -0.0022 & -0.0014 & -0.0006 & -0.0150 & -0.0156 & -0.0157 & 0.0050 & 0.0047 & 0.0038 \\
\hline 45 & -0.0025 & -0.0018 & -0.0008 & -0.0154 & -0.0158 & -0.0156 & 0.0046 & 0.0043 & 0.0036 \\
\hline 50 & -0.0024 & -0.0018 & -0.0009 & -0.0160 & -0.0168 & -0.0164 & 0.0042 & 0.0039 & 0.0039 \\
\hline
\end{tabular}

Averages over 20 independent agents starting from 20 randomly generated landscapes. The values reported represent the differences between the fitness values obtained by insensitive agents minus those obtained by the sensitive agents. Both types of agents are experienced new ventures. Insensitive agents do not adjust their confidence function in adopting protoroutines depending upon the observed profitability of the protoroutines (whereas sensitive ones do adjust). Bold values represent cases in which insensitive agents obtain higher performance relative to sensitive agents. SP represents the processing speed of the agent in terms of number of evaluations of potential mutations performed in a unit of time equal to 1. SD represents search distance. $K$ represents the level of complexity of the landscape. For each agent: $N=10, t_{0}=300, M=300$. 